\title{
Efeitos da menopausa nos ossos maxilares e na sobrevivência de implantes dentários
}

\author{
Effects of menopause on maxillary bones and dental implant survival \\ Efectos de la menopausia sobre los huesos maxilares y la supervivencia del implante dental
}

Recebido: 21/09/2021 | Revisado: 29/09/2021 | Aceito: 05/10/2021 | Publicado: 07/10/2021

\author{
Thaisa Renize Santos de Oliveira \\ ORCID: https://orcid.org/0000-0002-8213-7304 \\ Centro Universitário Mario Pontes Jucá, Brasil \\ E-mail: thaisarenize_deus@hotmail.com \\ Lucila Gabriela dos Santos \\ ORCID: https://orcid.org/0000-0003-4316-2627 \\ Centro Universitário Mario Pontes Jucá, Brasil \\ E-mail: lucila.santos126@ academico.fat-al.edu.br \\ Thiers Hendel Feitosa de Sales \\ ORCID: https://orcid.org/0000-0002-6143-9565 \\ Centro Universitário Mario Pontes Jucá, Brasil \\ E-mail: Thiersodonto@hotmail.com
}

\begin{abstract}
Resumo
O objetivo deste estudo foi analisar os efeitos do acometimento da osteoporose em mulheres na pós-menopausa, bem como a sua influência na sobrevivência de implantes dentários nesse grupo de mulheres. A revisão de literatura foi realizada através de um levantamento bibliográfico de estudos publicados nos últimos anos nas bases de dados da PubMed/Medline, Scopus, LILACS e SciELO. Os resultados informaram que a osteoporose afeta diretamente os ossos maxilares, causando modificações na sua qualidade e densidade ósseas. Porém, essas modificações não afetaram a taxa de sobrevida de implantes dentários nesse grupo feminino, o que também foi observado em relação ao uso de bifosfanos orais. É possível concluir a osteoporose causada pela menopausa causa mudanças ósseas nos maxilares, mas não afetam a sobrevivência de implantes dentários. O mesmo ocorre em mulheres que fazem uso de bifosfonatos orais.
\end{abstract}

Palavras-chave: Saúde da mulher; Osteoporose pós-menopausa; Implantes dentários; Bifosfonatos.

\begin{abstract}
Aim of this study was to analyze the effects of osteoporosis onset in postmenopausal women, as well as its influence on the survival of dental implants in this group of women. The literature review was carried out through a literature review of studies published in recent years in the databases of PubMed/Medline, Scopus, LILACS and SciELO. The results indicated that osteoporosis directly affects the maxillary bones, causing changes in their bone quality and density. However, these changes did not affect the survival rate of dental implants in this female group, which was also observed in relation to the use of oral bisphosphanes. It is possible to conclude that osteoporosis caused by menopause causes bone changes in the jaws, but does not affect the survival of dental implants. The same occurs in women who use oral bisphosphonates.
\end{abstract}

Keywords: Women's health; Osteoporosis postmenopausal; Dental implants; Biphosphonates.

\section{Resumen}

El objetivo de este estudio fue analizar los efectos de la aparición de la osteoporosis en mujeres posmenopáusicas, así como su influencia en la supervivencia de los implantes dentales en este grupo de mujeres. La revisión de la literatura se realizó a través de una revisión de la literatura de los estudios publicados en los últimos años en las bases de datos de PubMed / Medline, Scopus, LILACS y SciELO. Los resultados indicaron que la osteoporosis afecta directamente a los huesos maxilares, provocando cambios en su calidad y densidad ósea. Sin embargo, estos cambios no afectaron la tasa de supervivencia de los implantes dentales en este grupo de mujeres, lo que también se observó en relación con el uso de bisfosfanos orales. Es posible concluir que la osteoporosis causada por la menopausia provoca cambios óseos en los maxilares, pero no afecta la supervivencia de los implantes dentales. Lo mismo ocurre en mujeres que usan bisfosfonatos orales.

Palabras clave: Salud de la mujer; Osteoporosis mosmenopáusica; Implantes dentales; Bifosfonatos.

\section{Introdução}

A menopausa pode ser definida como a perda da atividade folicular dos ovários, acarretando supressão de sua função 
endócrina esteroidogênica. Essas mudanças ocasionam a cessação permanente da menstruação, sendo necessário um período de 12 meses de ausência menstrual para confirmar o diagnóstico desta condição. Esse período da vida da mulher inicia-se geralmente entre os 40 e 58 anos de idade, porém, inúmeros fatores podem afetar a idade inicial de transição da menopausa, como o índice de massa corporal (IMC), histórico familiar, etnia, menarca, número de partos e uso prévio de anticoncepcionais orais. (Mutneja, et al., 2012).

As mulheres na pós-menopausa apresentam diversos sintomas físicos e psicológicos decorrentes das mudanças hormonais durante esse período. A osteoporose é uma das manifestações mais relevantes encontradas dentro da literatura, sendo conceituada como um distúrbio esquelético sistêmico caracterizado pela perda da densidade óssea e alta suscetibilidade a fraturas ósseas. Seu advento durante o curso da menopausa é explicado pela diminuição dos níveis de estrogênio, que acarreta uma redução severa da massa óssea, intitulada de osteoporose pós-menopausa. (Puspitadewi et al., 2019).

Esse distúrbio torna as mulheres mais predispostas a desenvolver essa alteração nos ossos maxilares, ocasionando perda da altura óssea alveolar, sendo possível perder aproximadamente de 25 a $30 \%$ do osso cortical durante o período pósmenopausa. Isso pode resultar em perda de elementos dentários, prejudicando as funções estruturais do sistema estomatognático, e promovendo a desarmonia estética da população acometida por esse distúrbio. (Gaetti-Jardim et al., 2011).

Em casos de perda dentária, uma das melhores alternativas para reabilitação oral é o uso de implantes dentários, pois possuem excelentes resultados estéticos e funcionais. Todavia, um fator crítico para que se tenha um bom prognóstico após a instalação dos implantes é a ocorrência de uma alta taxa de osseointegração. Esse fator é influenciado diretamente pela qualidade e quantidade óssea do local de implante, que, como visto anteriormente, é alterado pela deficiência de estrogênio durante a menopausa, aumentando assim as chances de falha nos implantes dentários de mulheres na pós-menopausa (GaettiJardim et al., 2011; Ko et al., 2020). Buscando solucionar essas complicações, terapias com estrogênio podem evitar a reabsorção óssea dos maxilares, diminuindo os casos de falhas de implantes. (Mutneja, et al., 2012).

Perante o exposto, este trabalho objetiva avaliar os efeitos da menopausa na longevidade de implantes dentários, atentando-se aos impactos provocados pela osteoporose pós-menopausa e pela terapia com bifosfonatos na qualidade óssea dos maxilares.

\section{Metodologia}

Esta revisão de literatura integrativa foi elaborada com base em um checklist adaptado das diretrizes do PRISMA, tomando como referência etapas essenciais e bem definidas que visam estabelecer uma estruturação de qualidade para a revisão. (Moher et al., 2009; Hermont et al., 2021). Dessa forma, os critérios de inclusão adotados para a seleção dos artigos foram: estudos publicados entre janeiro de 2010 e janeiro de 2021 na língua portuguesa, inglesa e espanhola; trabalhos com populações de mulheres em idade compatível com o período de menopausa, que necessitem ou tenham realizado cirurgias para colocação de implantes dentário, bem como pacientes que fazem uso de medicação específica para diminuir os efeitos da menopausa (estrogênio e bifosfonatos). Já os critérios de exclusão dizem respeito a estudos não disponíveis integralmente, bem como aqueles realizados com mulheres acometidas por doenças crônicas, com histórico de doenças ósseas, que façam uso de medicações que afetam o metabolismo ósseo (como corticosteróides), presença de desordens metabólicas ou endócrinas ou que tenham realizado procedimentos de aumento ósseo durante as cirurgias de colocação dos implantes dentários.

A busca eletrônica foi feita com base nos seguintes descritores DeCS/ MeSH e operadores booleanos: "Menopause" AND "Dental implants" AND "Dentistry". A estratégia anterior foi aplicada nas seguintes bases de dados da área da saúde: PubMed/Medline, Scopus, LILACS e SciELO. Com base nisso, o levantamento bibliográfico foi elaborado a partir da leitura dos títulos e resumos dos estudos encontrados, selecionando aqueles que atenderem os critérios estabelecidos anteriormente. 
Após a seleção inicial, foi feita uma pesquisa manual das referências dos estudos, excluindo aqueles duplicados e os que não estavam disponíveis na íntegra para leitura. Os estudos selecionados foram por fim analisados com o auxílio de uma tabela, buscando diminuir os erros de transcrição das informações de cada estudo escolhido.

\section{Resultados e Discussão}

Foram encontrados 72 artigos ao todo através da busca eletrônica inicial. Após a leitura dos títulos e dos resumos, esse número reduziu para 11, sendo excluído 01 artigo por estar fora dos critérios estabelecidos para esse estudo, restando 10 artigos finais. Foram adicionados mais 02 artigos através da busca manual das referências dos estudos encontrados, contando com um total de 12 artigos finais. As etapas de seleção podem ser observadas no fluxograma abaixo (Figura 1), adaptado a partir das diretrizes do PRISMA.

Figura 1 - Fluxograma adaptado do PRISMA statement for systematic reviews.
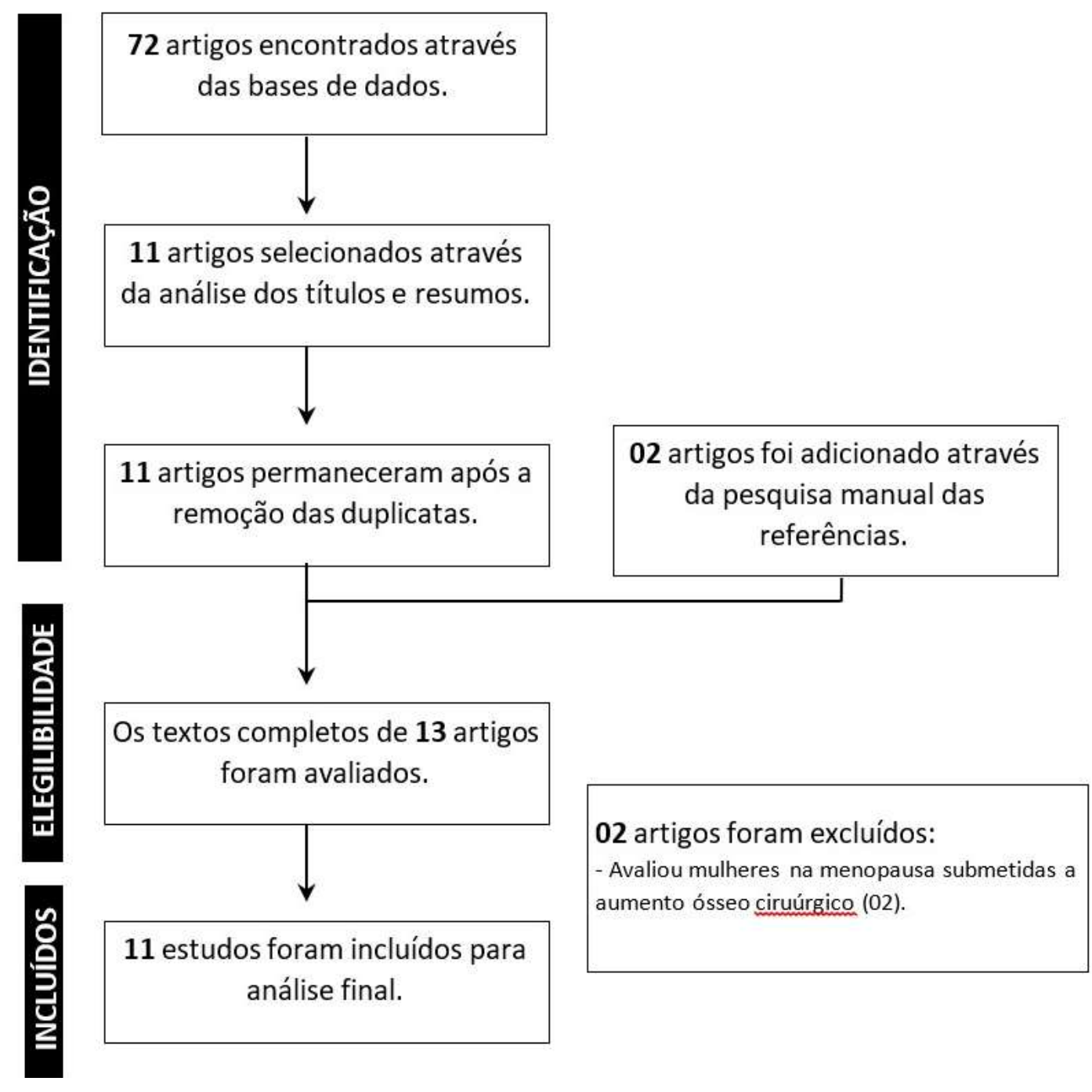

Fonte: Moher et al. (2009).

Um resumo ilustrando os principais achados, objetivos e conclusões dos artigos selecionados pode ser visto na tabela 1 deste estudo. O primeiro estudo, que pertence a Brasileiro et al. (2017), analisou os índices radiométricos de imagens por 
tomografia computadorizada de feixe cônico (TCFC) e índices de Densidade Mineral Óssea (DMO) em mulheres no período da pós-menopausa. Seguindo os índices de DMO, as mulheres incluídas no estudo foram divididas em três grupos: com osteopenia, osteoporose e normais. Observou-se que as mulheres com idade mais elevada eram aquelas que possuíam baixa DMO e que faziam parte dos grupos de osteopenia e osteoporose. Nas TCFC foram avaliados três índices que levavam em consideração a largura da cortical inferior óssea da mandíbula. Os resultados mostraram que esses índices eram estatisticamente menores em mulheres do grupo com osteoporose, quando comparado aos outros dois grupos. Também se observou que dois desses índices exibiram valores significativamente menores no grupo de mulheres com osteopenia, quando comparado ao grupo de mulheres normais.

O trabalho de Munakata et al. (2011), por sua vez, investigou o efeito da menopausa na quantidade e qualidade do osso mandibular na região edêntula de molares em mulheres que receberam implantes dentários. Isso foi feito através dos índices de DMO e pela medição das larguras da região do osso trabecular (OT) e osso cortical (OC) mandibulares com tomografias computadorizadas. Os valores de DMO foram consideravelmente baixos em mulheres na pós-menopausa. Já os valores das larguras das regiões de OT e OC variaram, apresentando-se significativamente maiores no OT e menores no OC em mulheres na pós-menopausa, sugerindo um percentual reduzido da cortical óssea nesse grupo de mulheres. Dessa forma, a DMO se correlacionou negativamente com a região de OT e positivamente com a de OC em ambos os grupos de mulheres na pré ou pós-menopausa.

Em seguida, o estudo de Iwasaki et al. (2011) teve como objetivo determinar se existe uma relação entre mudanças na densidade mineral óssea (DMO) na coluna lombar e no colo do fêmur e a perda dentária em mulheres pós-menopáusicas japonesas. Os resultados mostraram que o número médio de dentes perdidos durante o estudo foi de 1,2 e os valores da DMO declinaram 0,5 (1,4\%) na coluna lombar e 1,4 (0,9\%) no colo do fêmur por ano durante o período do estudo.

Posteriormente, Zhang et al. (2016) comparou a espessura óssea vestibular de dentes na zona estética de mulheres na pré-menopausa, pós menopausa, homens jovens e mais velhos. A espessura foi medida em milímetros em pontos de referência em incisivos centrais e laterais superiores e inferiores utilizando um software. Os resultados apontaram que os valores na espessura óssea dos locais medidos em mulheres na pós-menopausa diferiram dos valores do grupo de mulheres na prémenopausa e no de homens mais velhos. Mesmo quando as cristas ósseas médias foram comparadas no lugar dos locais específicos, as mulheres na pós-menopausa também exibiram valores diferentes entre os grupos da pré-menopausa e de homens mais velhos, sendo que os valores encontrados em mulheres na pós-menopausa foram mais baixos em relação aos demais grupos. Em contrapartida, os valores no grupo de mulheres na pré-menopausa não mostraram diferenças quando comparados aos grupos de homens mais jovens ou o de homens mais velhos.

Dvorak et al. (2011) avaliou a relação entre a osteoporose em mulheres na pós-menopausa e a taxa de sucesso de implantes dentários nessa população através de um exame clínico, utilizando como parâmetro a presença de peri-implantite nos locais com implantes dentários. Os resultados informaram que $26 \%$ das mulheres estudadas tinham osteoporose, $9 \%$ possuíam osteopenia e $65 \%$ não mostravam alterações na densidade óssea. A presença de peri-implantite foi registrada em 13,3\% dos implantes dentários avaliados e em $23,7 \%$ dos pacientes. $13,6 \%$ das pacientes tiveram perda tardia dos implantes dentários, dentre os quais as mulheres saudáveis possuíram perda tardia em 13\% dos casos, e as mulheres com osteopenia e osteoporose tiveram perda tardia em 18,75\% e 13\% dos casos, respectivamente. Já a perda do implante logo após a conexão do abutment foi vista em apenas $8,3 \%$ da população, correspondendo a uma taxa de 91,7\% de sobrevivência desses implantes.

Recentemente, Temmerman et al. (2019) estudou o desempenho clínico de implantes dentários em pacientes com diagnóstico de osteoporose/ osteopenia primária sistêmica, acarretado por alterações hormonais na menopausa. Desse modo, foram colocados implantes em pacientes com osteoporose, com idade variando de 59 a 83 anos, bem como em pacientes 
saudáveis, com idade entre 60 e 74 anos, realizando o acompanhamento desses implantes por 5 anos. Após esse período de tempo, somente 1 paciente do grupo com osteoporose sofreu perda de 5 implantes dentários, sendo a taxa de sobrevida após os 5 anos de $96,5 \%$ de toda a população do estudo. Essa população foi submetida a exames por tomografia computadorizada, onde foram obtidos os resultados dos níveis ósseos marginais, onde não se observou diferenças significativas acerca das alterações desses níveis ao longo dos 5 anos entre o grupo de pacientes com osteoporose e o grupo de pacientes saudáveis. Por fim, também foi realizado um levantamento clínico afim de determinar a presença de doença peri-implantar, que não apresentou diferenças ao longo do acompanhamento feito pelo estudo.

Mais tarde, Toy e Uslu (2020) também investigaram os efeitos da osteoporose/ osteopenia no sucesso a longo prazo e na perda óssea marginal de implantes dentários em mulheres com mais de 50 anos na pós-menopausa, sendo a média de idade da população estudada de 60,61 anos. Para tal objetivo, além da tomada dos níveis ósseos marginais, também foi realizado um exame clínico que estabeleceu o índice de placa, o estado do tecido mole peri-implantar através da profundidade de sondagem e sangramento a sondagem. Os resultados não mostraram diferenças estatisticamente relevantes entre os índices avaliados no exame clínico, bem como nos níveis ósseos marginais entre o grupo de mulheres com osteoporose e o grupo saudável, apesar de a média desses níveis ósseos terem sido um pouco maiores no grupo com osteoporose. Apenas o índice de sangramento gengival possuiu diferença estatisticamente significante entre os grupos, sendo maior nas mulheres com osteoporose (70,57\%) quando comparadas às saudáveis $(54,92 \%)$.

No mesmo ano, Ko et al. (2020) avaliaram a associação entre a menopausa e a espessura da cortical óssea crestal no local do implante dentário em uma população feminina através de TCFC. Essa população foi composta por 85 mulheres com idade média de 46 anos, que foi dividida em dois grupos: pacientes com idade inferior à média da menopausa (menos de 50 anos) e aqueles mais velhos do que a idade média da menopausa (acima de 50 anos). O grupo mais jovem mostrou espessura média da cortical crestal maior na mandíbula posterior $(1,29 \mathrm{~mm})$, seguido pela mandíbula anterior $(1,13 \mathrm{~mm})$, maxila anterior $(0,89 \mathrm{~mm})$ e maxila posterior $(0,7 \mathrm{~mm})$. De modo semelhante, no grupo mais velho, a espessura média da cortical crestal foi maior na mandíbula posterior $(1,27 \mathrm{~mm})$, seguido pela mandíbula anterior $(1,08 \mathrm{~mm})$, maxila anterior $(0,85 \mathrm{~mm})$ e maxila posterior $(0,24 \mathrm{~mm})$. Esses resultados demonstraram que o grupo de mulheres mais velhas obtiveram uma espessura média mais fina do osso cortical crestal, quando comparado ao grupo das mais novas, porém, essas diferenças só foram estatisticamente relevantes na região posterior da maxila (14,3\%) entre esses dois grupos.

Por sua vez, Yamashita-Mikami et al. (2013) examinou alterações microestruturais no osso esponjoso, decorrentes das alterações do metabolismo ósseo causadas pela menopausa, utilizando imagens de tomografia computadorizada de amostras de biópsias do osso alveolar extraído durante as cirurgias de colocação dos implantes dentários. O estudo foi composto por mulheres, que foram divididas em três grupos distintos: um grupo de pré-menopausa (entre 23 e 48 anos; n= 5), um de pósmenopausa precoce (entre 52 e 58 anos; $n=3$ ) e um grupo de pós-menopausa tardia (entre 60 e 75 anos; $n=10$ ), onde foram avaliados os marcadores de remodelação óssea, e, consequentemente, a atividade metabólica óssea sistêmica. Os resultados demonstraram o grupo pré-menopausa possuía o osso esponjoso altamente conectivo, com trabéculas interconectadas e espessas, diferentemente dos grupos pós-menopausa, onde o osso esponjoso se apresentou com baixa conectividade e trabéculas finas, dispersas e desconectadas. Os marcadores de remodelação óssea foram fortemente relacionados à morfologia trabecular dos ossos, pois à medida em que a formação óssea e a atividade de reabsorção aumentam com a renovação metabólica sistêmica dos ossos, as trabéculas ósseas se alteram morfologicamente.

Já o estudo de Koka et al. (2010) verificou se a sobrevivência de implantes dentários e a incidência de osteonecrose dos maxilares são as mesmas em pacientes com e sem histórico de uso de bifosfonatos. Para tal, foram avaliadas mulheres pósmenopáusicas com mais de 50 anos de idade que realizaram cirurgias para a colocação de implantes dentários, sendo 
classificadas em pacientes que tomam medicamentos com bifosfonatos para o tratamento de osteoporose ou osteopenia e que não fazem uso de tais medicamentos. Os resultados obtidos informaram que as taxas de sobrevivência dos implantes foram muito altas em ambos os grupos (99,17\% no grupo BP e 98,19\% no grupo sem BP), demonstrando que implantes dentários em pacientes que fazem uso de bifosfonatos não depende da utilização da medicação específica e exibem taxas de sobrevivência semelhante aos pacientes que não fazem uso dos bifosfonatos. Também foi possível observar que nenhum caso de osteonecrose dos maxilares ocorreu como resultado dos procedimentos cirúrgicos feitos ao longo da colocação dos implantes dentários.

Por fim, Yajima et al. (2017) avaliaram o efeito dos bifosfonatos orais nos ossos da mandíbula e as taxas de falhas de implantes em 25 pacientes do sexo feminino com osteoporose, que tinham mais de 60 anos de idade e tivessem sido tratadas com cirurgias de implantes dentários na região posterior da mandíbula. Dessa forma, a população foi dividida no grupo dos bifosfonatos e no grupo sem bifosfonatos. Nenhuma das pacientes avaliadas desenvolveu osteonecrose dos maxilares em nenhum dos dois grupos. No grupo BP ocorreram falha em 3 implantes dentários $(11,1 \%)$ de 3 pacientes distintos (25\%) em um ano. Já no grupo sem BP nenhum implante falhou ao longo de um período médio de acompanhamento de 5,2 anos. Mesmo com esses achados, não foram observadas diferenças estatisticamente relevantes entre os dois grupos de estudo. No que concerne à densidade mineral óssea (DMO) corticais e esponjosas, os valores encontrados no grupo BP foram maiores do que o outro grupo, porém, não foi estatisticamente significante, e não foram encontradas correlações entre os entre a duração de uso de bifosfonatos e as DMO corticais ou esponjosas. Também não houve diferença significativa na espessura do osso cortical entre ambos os grupos, apesar de que o uso prolongado dessa substância tende a contribuir para o aumento da espessura dos ossos corticais.

Tabela 1 - Características e dados dos artigos selecionados.

\begin{tabular}{|c|c|c|c|}
\hline Autor/ Ano & Amostra & Principais dados encontrados & Conclusões obtidas \\
\hline $\begin{array}{l}\text { Brasileiro et } \\
\text { al., } 2017\end{array}$ & $\begin{array}{l}\text { Mulheres entre } 47 \text { e } 78 \\
\text { anos divididas } \quad \text { em } 3 \\
\text { grupos: } \quad \text { pacientes } \\
\text { normais, }\end{array}$ & $\begin{array}{l}\text { Os grupos de osteopenia e osteoporose (maiores } \\
\text { médias de idade) tinham baixa DMO } \\
\text { (Densidade Mineral Óssea) quando comparados } \\
\text { ao grupo normal. Os índices da cortical inferior } \\
\text { mandibular foram menores nos grupos com } \\
\text { osteoporose em comparação com os demais. Já } \\
\text { o grupo osteopenia apresentou números } \\
\text { menores quando comparado ao grupo normal. }\end{array}$ & $\begin{array}{l}\text { Os índices avaliados são úteis na } \\
\text { identificação de mulheres na pós- } \\
\text { menopausa com baixa DMO e no } \\
\text { rastreamento para osteoporose. } \\
\text { Auxiliam os dentistas na triagem de } \\
\text { mulheres com baixa DMO para } \\
\text { encaminhá-las para densitometria } \\
\text { óssea. }\end{array}$ \\
\hline $\begin{array}{l}\text { Munakata } \\
\text { et al., } 2011\end{array}$ & $\begin{array}{l}72 \text { mulheres entre } 40 \text { e } \\
78 \text { anos, com região de } \\
\text { molar parcialmente } \\
\text { edêntula da mandíbula } \\
\text { para receber implantes } \\
\text { dentários. } 30 \text { estavam } \\
\text { na pré-menopausa e } 42 \\
\text { na pós-menopausa. }\end{array}$ & $\begin{array}{l}\text { Mulheres na pós-menopausa apresentaram } \\
\text { valores na DMO mais baixas. Valores acerca da } \\
\text { largura da região do osso trabecular (OT) foram } \\
\text { maiores nas mulheres na pós-menopausa e } \\
\text { menores na do osso cortical (OC). A DMO se } \\
\text { correlacionou positivamente com o OC e } \\
\text { negativamente com o OT em mulheres na pré } \\
\text { ou pós-menopausa. }\end{array}$ & $\begin{array}{l}\text { A medida quantitativa da densidade } \\
\text { óssea é útil para o tratamento de } \\
\text { implantes dentários em mulheres na } \\
\text { pós-menopausa. }\end{array}$ \\
\hline $\begin{array}{ll}\text { Iwasaki } & \text { et } \\
\text { al., } 2011 & \end{array}$ & $\begin{array}{l}404 \text { mulheres na pós- } \\
\text { menopausa com idade } \\
\text { média de } 64.2 \text { anos. }\end{array}$ & $\begin{array}{l}\text { O número médio de dentes perdidos foi de } 1,2 \text { e } \\
\text { os valores da DMO declinaram } 1,4 \% \text { na coluna } \\
\text { lombar e } 0,9 \% \text { no colo do fêmur por ano. }\end{array}$ & $\begin{array}{l}\text { As alterações da DMO foram } \\
\text { associadas ao número de dentes } \\
\text { perdidos em mulheres japonesas pós- } \\
\text { menopáusicas. }\end{array}$ \\
\hline $\begin{array}{l}\text { Zhang et al., } \\
2016\end{array}$ & $\begin{array}{l}60 \quad \text { prontuários } \\
\text { dividindo os pacientes } \\
\text { em: mulheres na pré- } \\
\text { menopausa, pós- } \\
\text { menopausa, homens } \\
\text { mais jovens e homens }\end{array}$ & $\begin{array}{l}\text { Os grupos de mulheres na pré-menopausa, na } \\
\text { pós-menopausa e o de homens mais velhos } \\
\text { diferiram quando a espessura óssea dos locais } \\
\text { medidos e das médias das cristas ósseas. Já as } \\
\text { mulheres na pré-menopausa quando comparado } \\
\text { aos homens mais jovens e mais velhos não }\end{array}$ & $\begin{array}{l}\text { O osso na zona estética de dentes } \\
\text { anteriores é fino em todos os } \\
\text { pacientes, no entanto, o osso dessa } \\
\text { zona é significativamente mais fino } \\
\text { em mulheres na pós-menopausa. }\end{array}$ \\
\hline
\end{tabular}




\begin{tabular}{|c|c|c|c|}
\hline $\begin{array}{ll}\text { Dvorak } & \text { et } \\
\text { al., } 2011 & \end{array}$ & $\begin{array}{l}203 \text { mulheres com } 967 \\
\text { implantes sendo } \\
\text { classificadas em } \\
\text { saudáveis, com } \\
\text { osteopenia e } \\
\text { osteoporose. }\end{array}$ & $\begin{array}{l}26 \% \text { tinham osteoporose, } 9 \% \text { osteopenia e } 65 \% \\
\text { eram saudáveis. A presença de peri-implantite } \\
\text { foi registrada em } 13,3 \% \text { dos implantes e em } \\
23,7 \% \text { das pacientes. A perda tardia de } \\
\text { implantes dentários ocorreu em } 13,6 \% \text { e a perda } \\
\text { após a conexão do abutment em } 8,3 \% \text {. A taxa } \\
\text { de sobrevivência dos implantes foi de } 91,7 \% \text {. }\end{array}$ & $\begin{array}{l}\text { Dados sugerem que a osteoporose } \\
\text { pós-menopáusica não é um fator de } \\
\text { risco para a perda de implantes e para } \\
\text { a presença de peri-implantites. }\end{array}$ \\
\hline $\begin{array}{l}\text { Temmerma } \\
\text { n et al., } 2019\end{array}$ & $\begin{array}{l}148 \text { implantes de } 48 \\
\text { pacientes divididos em } \\
\text { dois grupos: com } \\
\text { osteoporose } \\
\text { saudáveis. }\end{array}$ & $\begin{array}{l}\text { Ocorreu perda de } 5 \text { implantes em } 1 \text { paciente. A } \\
\text { taxa de sobrevida dos implantes foi de } 96,5 \% \\
\text { após } 5 \text { anos de acompanhamento. Não houve } \\
\text { diferenças estatísticas entre os níveis ósseos } \\
\text { marginais e nem com relação a presença de } \\
\text { doença peri-implantar entre os dois grupos. }\end{array}$ & $\begin{array}{l}\text { A terapia com implantes dentários é } \\
\text { uma opção de tratamento viável em } \\
\text { pacientes com osteoporose, com taxa } \\
\text { de sobrevida semelhante aos pacientes } \\
\text { saudáveis do estudo. }\end{array}$ \\
\hline $\begin{array}{l}\text { Toy \& Uslu, } \\
2020\end{array}$ & $\begin{array}{l}280 \text { mulheres com mais } \\
\text { de } 50 \text { anos de idade } \\
\text { com pelo menos um } \\
\text { implante dentário, } \\
\text { sendo divididas em: } \\
\text { pacientes } \\
\text { osteoporose com } \\
\text { saudáveis. }\end{array}$ & $\begin{array}{l}\text { A taxa de sobrevivência dos implantes foi de } \\
98,1 \% \text {. Os índices clínicos não apresentaram } \\
\text { diferenças entre os grupos, com exceção ao } \\
\text { índice de sangramento a sondagem que foi } \\
\text { maior no grupo com osteoporose, com } 70,57 \% \\
\text { e 30,7\% no grupo saudável. Os níveis ósseos } \\
\text { marginais também não exibiram diferença entre } \\
\text { os grupos avaliados. }\end{array}$ & $\begin{array}{l}\text { A terapia com implantes dentários é } \\
\text { uma opção válida para pacientes com } \\
\text { diagnóstico de osteoporose/ } \\
\text { osteopenia, não sendo contraindicados } \\
\text { a esse grupo de pessoas. }\end{array}$ \\
\hline $\begin{array}{l}\text { Ko et al., } \\
2020\end{array}$ & $\begin{array}{l}85 \text { mulheres } \\
\text { submetidas à colocação } \\
\text { de implantes dentários, } \\
\text { divididas em } 2 \text { grupos: } \\
\text { com idade inferior à } \\
\text { média da menopausa e } \\
\text { com idade média da } \\
\text { menopausa. }\end{array}$ & $\begin{array}{l}\text { Ambos os grupos apresentaram espessura média } \\
\text { maior da cortical crestal na mandíbula posterior, } \\
\text { seguido da anterior, maxila anterior e posterior. } \\
\text { O grupo mais velho exibiu espessura média } \\
\text { óssea mais fina do as mais novas, sendo } \\
\text { significativamente relevante apenas na região } \\
\text { posterior da maxila }(14,3 \%) \text {. }\end{array}$ & $\begin{array}{l}\text { Para mulheres mais jovens e mais } \\
\text { velhas as regiões com maior e menor } \\
\text { espessura de osso cortical crestal são } \\
\text { a região posterior da mandíbula e a } \\
\text { posterior da maxila, respectivamente. } \\
\text { Além disso, as mulheres mais velhas } \\
\text { possuem a cortical óssea mais fina } \\
\text { que as mais novas. }\end{array}$ \\
\hline $\begin{array}{l}\text { Yamashita- } \\
\text { Mikami et } \\
\text { al., } 2013\end{array}$ & $\begin{array}{l}18 \text { mulheres japonesas } \\
\text { dividas em } 3 \text { grupos: } \\
\text { pré-menopausa (entre } \\
23 \text { e } 48 \text { anos), pós- } \\
\text { menopausa precoce } \\
\text { (entre } 52 \text { e } 58 \text { anos) e } \\
\text { pós-menopausa tardia } \\
\text { (entre } 60 \text { e } 75 \text { anos). }\end{array}$ & $\begin{array}{l}\text { O grupo pré-menopausa possuía o osso } \\
\text { esponjoso altamente conectivo, com trabéculas } \\
\text { interconectadas e espessas, diferentemente dos } \\
\text { grupos pós-menopausa, onde o osso esponjoso } \\
\text { se apresentou com baixa conectividade e } \\
\text { trabéculas finas, dispersas e desconectadas. Os } \\
\text { marcadores de remodelação óssea foram } \\
\text { fortemente relacionados à morfologia trabecular } \\
\text { dos ossos. }\end{array}$ & $\begin{array}{l}\text { O osso esponjoso alveolar feminino } \\
\text { começa a se deteriorar logo após a } \\
\text { menopausa e a microestrutura do osso } \\
\text { esponjoso alveolar respondem } \\
\text { rapidamente às mudanças nos } \\
\text { marcadores de renovação óssea } \\
\text { sistêmica. }\end{array}$ \\
\hline $\begin{array}{l}\text { Koka et al., } \\
2010\end{array}$ & $\begin{array}{l}\text { Mulheres pós- } \\
\text { menopáusicas divididas } \\
\text { em } 2 \text { grupos: um das } \\
\text { que fazem uso de } \\
\text { bifosfonatos e outro } \\
\text { das que não fazem uso. }\end{array}$ & $\begin{array}{l}\text { As taxas de sobrevivência foram altas entre } \\
\text { ambos os grupos }(99,17 \% \text { no grupo que faz uso } \\
\text { de bifosfonatos e } 98,19 \% \text { nos grupos que não } \\
\text { faz uso das medicações). Implantes colocados } \\
\text { em usuários de bifosfonatos possuem perfis de } \\
\text { sobrevivência semelhante aos que não usam. }\end{array}$ & $\begin{array}{l}\text { Os implantes dentários colocados em } \\
\text { maxilares de mulheres na pós- } \\
\text { menopausa com histórico de uso de } \\
\text { bifosfonatos têm um excelente perfil } \\
\text { de sobrevida. Implantes dentários em } \\
\text { usuários de bifosfonatos são seguros. }\end{array}$ \\
\hline $\begin{array}{l}\text { Yajima } \\
\text { al., } 2017\end{array}$ & $\begin{array}{l}25 \text { mulheres com pelo } \\
\text { menos } 60 \text { anos de idade } \\
\text { divididas em } 2 \text { grupos: } \\
\text { grupo dos bifosfonatos } \\
\text { e grupo sem } \\
\text { bifosfonatos. }\end{array}$ & $\begin{array}{l}\text { Nenhum paciente desenvolveu osteonecrose dos } \\
\text { maxilares. A sobrevida dos implantes foi } \\
\text { semelhante em ambos os grupos. A DMO foi } \\
\text { maior no grupo do bifosfonatos e não houve } \\
\text { diferença significativa entre os grupos quanto a } \\
\text { espessura do osso cortical. }\end{array}$ & $\begin{array}{l}\text { Os bifosfonatos podem afetar a } \\
\text { qualidade e a quantidade do osso } \\
\text { cortical na mandíbula posterior } \\
\text { parcialmente edêntula de pacientes } \\
\text { com osteoporose. }\end{array}$ \\
\hline
\end{tabular}

Fonte: Autores (2021).

Brasileiro et al., 2017 Mulheres entre 47 e 78 anos divididas em 3 grupos: pacientes normais, com osteopenia e com osteoporose. Os grupos de osteopenia e osteoporose (maiores médias de idade) tinham baixa DMO (Densidade Mineral Óssea) 
quando comparados ao grupo normal. Os índices da cortical inferior mandibular foram menores nos grupos com osteoporose em comparação com os demais. Já o grupo osteopenia apresentou números menores quando comparado ao grupo normal. Os índices avaliados são úteis na identificação de mulheres na pós-menopausa com baixa DMO e no rastreamento para osteoporose. Auxiliam os dentistas na triagem de mulheres com baixa DMO para encaminhá-las para densitometria óssea.

Munakata et al., 2011, 72 mulheres entre 40 e 78 anos, com região de molar parcialmente edêntula da mandíbula para receber implantes dentários. 30 estavam na pré-menopausa e 42 na pós-menopausa. Mulheres na pós-menopausa apresentaram valores na DMO mais baixas. Valores acerca da largura da região do osso trabecular (OT) foram maiores nas mulheres na pósmenopausa e menores na do osso cortical (OC). A DMO se correlacionou positivamente com o OC e negativamente com o OT em mulheres na pré ou pós-menopausa. A medida quantitativa da densidade óssea é útil para o tratamento de implantes dentários em mulheres na pós-menopausa.

Iwasaki et al., 2011, 404 mulheres na pós-menopausa com idade média de 64.2 anos. O número médio de dentes perdidos foi de 1,2 e os valores da DMO declinaram 1,4\% na coluna lombar e $0,9 \%$ no colo do fêmur por ano. As alterações da DMO foram associadas ao número de dentes perdidos em mulheres japonesas pós-menopáusicas.

Zhang et al., 2016, 60 prontuários dividindo os pacientes em: mulheres na pré-menopausa, pós-menopausa, homens mais jovens e homens mais velhos. Os grupos de mulheres na pré-menopausa, na pós-menopausa e o de homens mais velhos diferiram quando a espessura óssea dos locais medidos e das médias das cristas ósseas. Já as mulheres na pré-menopausa quando comparado aos homens mais jovens e mais velhos não mostraram diferenças nas medições obtidas. O osso na zona estética de dentes anteriores é fino em todos os pacientes, no entanto, o osso dessa zona é significativamente mais fino em mulheres na pós-menopausa.

Dvorak et al., 2011, 203 mulheres com 967 implantes sendo classificadas em saudáveis, com osteopenia e osteoporose. $26 \%$ tinham osteoporose, $9 \%$ osteopenia e $65 \%$ eram saudáveis. A presença de peri-implantite foi registrada em $13,3 \%$ dos implantes e em $23,7 \%$ das pacientes. A perda tardia de implantes dentários ocorreu em 13,6\% e a perda após a conexão do abutment em 8,3\%. A taxa de sobrevivência dos implantes foi de 91,7\%. Dados sugerem que a osteoporose pósmenopáusica não é um fator de risco para a perda de implantes e para a presença de peri-implantites.

Temmerman et al., 2019148 implantes de 48 pacientes divididos em dois grupos: com osteoporose e saudáveis.

Ocorreu perda de 5 implantes em 1 paciente. A taxa de sobrevida dos implantes foi de 96,5\% após 5 anos de acompanhamento. Não houve diferenças estatísticas entre os níveis ósseos marginais e nem com relação a presença de doença peri-implantar entre os dois grupos. A terapia com implantes dentários é uma opção de tratamento viável em pacientes com osteoporose, com taxa de sobrevida semelhante aos pacientes saudáveis do estudo.

Toy \& Uslu, 2020280 mulheres com mais de 50 anos de idade com pelo menos um implante dentário, sendo divididas em: pacientes com osteoporose e saudáveis. A taxa de sobrevivência dos implantes foi de 98,1\%. Os índices clínicos não apresentaram diferenças entre os grupos, com exceção ao índice de sangramento a sondagem que foi maior no grupo com osteoporose, com 70,57\% e 30,7\% no grupo saudável. Os níveis ósseos marginais também não exibiram diferença entre os grupos avaliados. A terapia com implantes dentários é uma opção válida para pacientes com diagnóstico de osteoporose/ osteopenia, não sendo contraindicados a esse grupo de pessoas.

Ko et al., 202085 mulheres submetidas à colocação de implantes dentários, divididas em 2 grupos: com idade inferior à média da menopausa e com idade média da menopausa. Ambos os grupos apresentaram espessura média maior da cortical crestal na mandíbula posterior, seguido da anterior, maxila anterior e posterior. O grupo mais velho exibiu espessura média óssea mais fina do as mais novas, sendo significativamente relevante apenas na região posterior da maxila (14,3\%).

Para mulheres mais jovens e mais velhas as regiões com maior e menor espessura de osso cortical crestal são a região 
posterior da mandíbula e a posterior da maxila, respectivamente. Além disso, as mulheres mais velhas possuem a cortical óssea mais fina que as mais novas.

Yamashita-Mikami et al., 201318 mulheres japonesas dividas em 3 grupos: pré-menopausa (entre 23 e 48 anos), pósmenopausa precoce (entre 52 e 58 anos) e pós-menopausa tardia (entre 60 e 75 anos). O grupo pré-menopausa possuía o osso esponjoso altamente conectivo, com trabéculas interconectadas e espessas, diferentemente dos grupos pós-menopausa, onde o osso esponjoso se apresentou com baixa conectividade e trabéculas finas, dispersas e desconectadas. Os marcadores de remodelação óssea foram fortemente relacionados à morfologia trabecular dos ossos. O osso esponjoso alveolar feminino começa a se deteriorar logo após a menopausa e a microestrutura do osso esponjoso alveolar respondem rapidamente às mudanças nos marcadores de renovação óssea sistêmica.

Koka et al., 2010 Mulheres pós-menopáusicas divididas em 2 grupos: um das que fazem uso de bifosfonatos e outro das que não fazem uso. As taxas de sobrevivência foram altas entre ambos os grupos $(99,17 \%$ no grupo que faz uso de bifosfonatos e 98,19\% nos grupos que não faz uso das medicações). Implantes colocados em usuários de bifosfonatos possuem perfis de sobrevivência semelhante aos que não usam. Os implantes dentários colocados em maxilares de mulheres na pósmenopausa com histórico de uso de bifosfonatos têm um excelente perfil de sobrevida. Implantes dentários em usuários de bifosfonatos são seguros.

Yajima et al., 2017, 25 mulheres com pelo menos 60 anos de idade divididas em 2 grupos: grupo dos bifosfonatos e grupo sem bifosfonatos. Nenhum paciente desenvolveu osteonecrose dos maxilares. A sobrevida dos implantes foi semelhante em ambos os grupos. A DMO foi maior no grupo do bifosfonatos e não houve diferença significativa entre os grupos quanto a espessura do osso cortical. Os bifosfonatos podem afetar a qualidade e a quantidade do osso cortical na mandíbula posterior parcialmente edêntula de pacientes com osteoporose.

Os efeitos do período da menopausa na saúde das mulher são amplamente discutidos, e incluem inúmeras mudanças sistêmicas no organismo dessas mulheres. Pensando nisso, um dos distúrbios altamente prevalentes que nesse período é o desenvolvimento de osteoporose, uma doença esquelética sistêmica caracterizada pela deterioração da microestrutura óssea, causando redução significativa da massa ósseoa, aumentando as chances de ocorrer fratura nos ossos. Quando a osteoporose é causada por mudanças ocorridas com a idade, como as alterações hormomais da menopausa, é chamada de osteoporose primária. Por ser uma doença sistêmica, ela afeta toda a estrutura esquelética do indivíduo, incluindo os ossos maxilares, que são bastante abordados em estudos científicos. Assim, dois fatores são fundamentais para determinar a resistência desses ossos: a densidade óssea, determinada pelo valor máximo da massa óssea; e a qualidade do osso, que depende da sua microestrutura, da remodelação, de microfraturas e o grau de mineralizaçao óssea. (Munakata et al., 2011; Mellado-Valero et al., 2010).

O diagnóstico da osteoporose primária é feito através da avaliação da densidade mineral óssea (DMO) através da densiometria óssea. A DMO é um dos parâmetros mais utilizados em estudos que avaliam a qualidade óssea de mulheres na pós-menopausa. Esses estudos mostram que os valores da DMO em maxilares são mais baixos em mulheres acometidas pela osteoporose quando comparado a mulheres saudáveis na menopausa, esses valores também tendem a decrescer com o passar dos anos nesses grupos de mulheres pós-menopáusicas. A espessura da cortical óssea dos maxilares é outro parâmetro utilizado para avaliar a qualidade dos ossos, e exibem valores menores em mulheres no período da pós-menopausa acometidas por osteoporose, quando comparado a mulheres saudáveis nesse mesmo período. (Nascimento et al., 2017; Munakata et al., 2011; Iwasaki et al., 2011). A respeito desse tema, Zhang et al. (2016) demonstrou que a espessura óssea em zonas dentárias estéticas difere entre mulheres na pós-menopausa e mulheres mais jovens, afirmando que a espessura diminui no período da menopausa.

Além diso, verifica-se também a ocorrência de alterações microestruturais no osso esponjoso devido a alterações do metabolismo ósseo causadas pela menopausa. Mulheres na pós-menopausa apresentam uma configuração óssea com trabéculas 
finas, dispersas e desconectadas. Já as mulheres mais jovens, fora da faixa etária média da menopausa possuem trabéculas ósseas mais espessas e interconectadas. Essas alterações na morfologia trabecular estão relacionadas ao aumento da formação e reabsorção ósseas, que intensificam a renovação metabólica sistêmica dos ossos, permitindo visualizar essas diferenças entre os grupos de mulheres. (Yamashita-Mikami et al., 2013).

Ao afetar os ossos maxilares, a osteoporose pode causar muitos problemas na cavidade oral, sendo um deles associado

à perda de elementos dentários. É possível observar uma associação relevante entre as mudanças na DMO e o número de dentes perdidos em mulheres na pós-menopausa. Isso ocorre pelo fato de que as mulheres com valores mais baixos na DMO mostraram ter maior probabilidade de perder dentes. (Iwasaki et al., 2011).

Uma outra questão odontológica que está sendo amplamente estudada diz respeito à longevidade de implantes dentários presentes em mulheres pós-menopáusicas. Estudos mostram que mulheres na pós-menopausa e aquelas que apresentam osteoporose possuem taxas muito altas de sobrevivência de seus implates ao londo do tempo (acima de 90\%). A presença de doença pri-implantar é um fator determinante nesse processo. Entretanto, os dados afirmam que não há diferenças estatisticamente significantes entre o aparecimento de peri-implantites ao longo dos anos, independente de serem mulheres na pós-menopausa on não. (Toy e Uslu, 2020; Temmerman et al., 2019; Dvorak et al. 2011). Apesar dos estudos demonstrarem que a menopausa não afeta diretamente a estabilidade de implantes dentários, a espessura da cortical óssea crestal em locais de implantes dentários sofre modificações de acordo com a idade das mulheres, sendo que as mais velhas apresentaram espessura média mais fina do osso cortical, principalmente na região posterior da maxila, quando comparadas a mulheres mais jovens, o que está diretamente ligado aos efeitos da menopausa na característica óssea desses indivíduos. (Ko et al., 2020).

Suspeita-se ainda que algumas medicações usadas para diminuir esses efeitos, e em especial aqueles utilizados par ao controle da osteoporose primária podem induzir a diminuição da sobrevivência dos implantes dentários. Em se tratando de controlar a osteoporose, um dos principais tratamentos empregados para essa finalidade é uso de bifosfonatos orais. O seu uso por tempo prolongado está ligado ao surgimento osteonecrose dos maxilares, que consiste em focos de osso necrótico com exposição do osso maxilar ou mandibular. Apesar da associação, os estudos demonstraram que a taxa de sobrevivência de implantes em mulheres que faziam uso dessa medicação era semelhante àquelas que não faziam uso, e que as falhas relatadas dos implantes avaliados não podiam ser atribuídas aos bifosfonatos, bem como não foram observados casos de osteonecrose dos maxilares nas populações estudadas. Dessa forma, os bifosfonatos podem afetar a qualidade e quantidade óssea, mas os procedimentos de colocação de implantes dentários em mulheres que fazem uso dessa substância são considerados seguros. (Koka et al., 2010; Yajima et al., 2017).

\section{Conclusão}

A ocorrência de osteoporose em mulheres na pós-menopausa afeta diretamente os ossos maxilares, causando diminuição da DMO, alterações na largura do osso cortical, bem como na microestrutura desses ossos. A osteoporose também foi associada a perda de elementos dentários. Mesmo com as modificações ósseas, o tratamento com implantes dentários é considerado seguro para as mulheres com menopausa, pois os estudos exibiram alta taxa de sobrevivência dos implantes ao longo dos anos. O uso de bifosfonatos não foi correlacionado a falhas e perdas de implantes dentários nesse grupo de mulheres.

Apesar dos achados promissores, novos estudos referentes a terapia hormonal com um número amostral efetivamente maior é importante para reforçar o nível de evidência científica. 


\section{Referências}

Brasileiro, C. B., Chalub, L. L. F. H., Abreu, M. H. N. G., Barreiros, I. D., Amaral, T. M. P., Kakehasi, A. M., \& Mesquita, R. A. (2017). Use of cone beam computed tomography in identifying postmenopausal women with osteoporosis. Arch Osteoporos, 12 (1), 1-6.

Dvorak, G., Arnhart, C., Heuberer, S., Huber, C. D., \& Watzek, G. (2011). Peri-implantitis and late implant failures in postmenopausal women: a crosssectional study. J Clin Periodontol, 38 (1), 950-955.

Gaetti-Jardim, E. C., Santiago-Junior, J. F., Goiato, M. C., Pelizer, E. P., Magno-Filho, O., \& Jardim-Junior, E. G. (2011). Dental Implants in Patients With Osteoporosis. Journal Of Craniofacial Surgery, 22 (3), 1111-1113.

Hermont, A. P., Zina, L. G., Silva, K. D., Silva, J. M., \& Martins-Júnior, P. A. (2021). Revisões integrativas: conceitos, planejamento e execução. Arq Odontol, 57 (1), 3-7.

Iwasaki, M., Nakamura, K., Yoshihara, A., \& Miyazaki, H. (2011). Change in bone mineral density and tooth loss in Japanese community-dwelling postmenopausal women: a 5-year cohort study. J Bone Miner Metab, 30 (1), 447-453.

Ko, Y., Tsai, M., Fuh, L., Wang, X., Huang, H., \& Hsu, J. (2020). Association between Age of Menopause and Thickness of Crestal Cortical Bone at Dental Implant Site: a cross-sectional observational study. International. Journal Of Environmental Research And Public Health, 17 (5868), 1-9.

Koka, S., Babu, N. M. S., \& Norell, A. (2010). Survival of dental implants in post-menopausal bisphosphonate users. Journal of Prosthodontic Research, 54 (1), 108-111.

Mellado-Valero, A., Ferrer-Garcia, J. C., Calvo-Catalá, J., \& Labaig-Rueda, C. (2010). Implant treatment in patients with osteoporosis. Med Oral, Patol Oral and Cir Bucal, 15 (1), 52-57.

Moher, D., Liberati, A., Tetzlaff, J., Altman, D. G., \& The PRISMA Group. (2009). Preferred Reporting Items for Systematic Reviews and Meta-Analyses: The PRISMA Statement. Plos Medicine, 6 (7), 1-6.

Munakata, M., Tachikawa, N., Honda, E., Shiota, M., \& Kasugai, S. (2011). Influence of menopause on mandibular bone quantity and quality in Japanese women receiving dental implants. Arch Osteoporos, 6 (1), 51-57.

Mutneja, P., Dhawan, P., Raina, A., \& Sharma, G. (2012). Menopause and the oral cavity. Indian Journal Of Endocrinology And Metabolism, 16 (4), 548-551.

Puspitadewi, S. R., Wulandari, P., Kusdhany, L. S., Masulili, S. L. C., Iskandar, H. B., \& Auerkari, E. I. (2019). Relationship of Age, Body Mass Index, Bone Density, and Menopause Duration with Alveolar Bone Resorption in Postmenopausal Women. Pesquisa Brasileira em Odontopediatria e Clínica Integrada, $19(1), 1-10$.

Temmerman, A., Rasmusson, L., Kubler, A., Thor, A., Merheb, J., \& Quirynen, M. (2019). A Prospective, Controlled, Multicenter Study to Evaluate the Clinical Outcome of Implant Treatment in Women with Osteoporosis/Osteopenia: 5-Year Results. Journal of Dental Research, 98 (1), 84-90.

Toy, V. E., \& Uslu, M. O. (2020). Evaluation of Long-Term Dental Implant Success and Marginal Bone Loss in Postmenopausal Women. Niger J Clin Pract, $23(1), 147-153$.

Yajima, N., Munakata, M., Fuchigami, K., Sanda, M., \& Kasugai, S. (2017). Influence of Bisphosphonates on Implant Failure Rates and Characteristics of Postmenopausal Woman Mandibular Jawbone. Journal of Oral Implantology, 42 (5), 345-349.

Yamashita-Mikami, E., Tanaka, M., Sakurai, N., Arai, Y., Matsuo, A., Ohshima, H., Nomura, S., \& Ejiri, S. (2013). Correlations between alveolar bone microstructure and bone turnover markers in pre- and post-menopausal women. Oral Surg, Oral Med, Oral Pathol and Oral Radiol, 115 (4), e12-e19.

Zhang, C. Y., DeBaz, C., Bhandal, G., Alli, F., Francisco, M. C. B., Thacker, H. L., Palomo, J. M., \& Palomo, L. (2016). Buccal Bone Thickness in the Esthetic Zone of Postmenopausal Women: A CBCT Analysis. Implant Dentistry, 25 (4), 478-484. 